\title{
Magnetoroton scattering by phonons in the fractional quantum Hall régime
}

\author{
KA Benedict and RK HIlls \\ School of Physics and Astronomy, \\ The University of Nottingham \\ University Park \\ NOTTINGHAM \\ NG7 2RD \\ U.K.
}

\begin{abstract}
Motivated by recent phonon spectroscopy experiments in the fractional quantum Hall regime we consider processes in which thermally excited magnetoroton excitations are scattered by low energy phonons. We show that such scattering processes can never give rise to dissociation of magnetorotons into unbound charged quasiparticles as had been proposed previously. In addition we show that scattering of magnetorotons to longer wavelengths by phonon absorption is possible because of the shape of the magnetoroton dispersion curve and it is shown that there is a characteristic cross-over temperature above which the rate of energy transfer to the electron gas changes from an exponential (activated) to a power law dependence on the effective phonon temperature.
\end{abstract}

\section{Introduction}

Phonon spectroscopy is a technique that has been used with much success in the study of semiconductor systems 11 and in particular the properties of twodimensional electron systems (2des) formed at heterojunctions and quantum wells. One of the most important recent applications of the technique has been to the study of the incompressible quantum liquid of electrons formed at a semiconductor heterojunction which gives rise to the fractional quantum Hall effect 2, 3]. The essential points of the experiments are that a pulse of phonons with an approximately black body spectrum of energies is injected into the substrate of a semiconductor device. The phonons propagate ballistically across the substrate until they encounter the electrons at the heterojunction where some of them may be absorbed. In the fractional quantum Hall states the electrons have a true energy gap as a consequence of incompressibility, so that if the electrons are initially at zero temperature, only the phonons in the high energy 
part of the black body distribution can be absorbed. It is a great virtue of the technique that these phonons may also have wave vectors comparable with the interesting structure in the dispersion relation predicted for the low lying collective modes or magnetorotons [4] of the electron liquid. The basic theoretical picture of what happens in these experiments has been outlined recently [5]. This work concentrated on processes in which the absorption of a high energy phonon leads to the creation of a magnetoroton excitation close to the minimum in the dispersion curve. In the experiments, the effect of the phonon absorption is detected by measuring changes in the two-terminal resistance of the device. It was speculated [2] that in some way the elementary excitations created by the absorption of phonons contributed more or less directly to the increased dissipation observed in the two-terminal measurements. Dissipation due to mutual friction between the magnetoroton gas and the Laughlin liquid has been discussed by Platzman [6]. A more concrete mechanism for dissipation due to magnetoroton creation was proposed by one of us [7]. The essential idea of this was that, once created by absorption of a high-energy phonon, a magnetoroton excitation would rapidly absorb many low-energy phonons from the bulk of the Planck distribution in the pulse. For wave vectors larger than $q^{*}$ the magnetorotons can be viewed as excitons formed from Laughlin quasiparticles and quasiholes [8], the separation of the two quasiparticles in an exciton with wave vector $q$ being $l_{c}^{2} q$. Once an exciton has absorbed sufficient low energy phonons that the separation of its constituents exceeds some effective screening length, the fractionally charged quasiparticles are effectively unbound and contribute directly to the two-terminal resistance. A crude analysis presented in [7] indicated that this process might not be particularly rapid. One purpose of this paper is to analyse this idea more carefully and in fact to show that the process described will not actually happen at all. The shape of the magnetoroton dispersion curve is such that the processes in which magnetorotons absorb energy from the low-energy rump of the Planck distribution will always reduce the wave vector of the magnetoroton. Hence we conclude, as discussed in [5], that the energy absorbed from the phonon pulse by the electrons is shared among all the electrons leading to an overall increase in the electron temperature, which then leads to an increased two-terminal resistance for the device. The processes described above in which magnetorotons are heated with a corresponding decrease in wave vector will, of course, occur in real experiments and will provide the leading correction to the expressions described in [5] at finite electron temperature. These corrections are of particular importance for recent experiments [3] in which the time dependence of the two-terminal resistance, and therefore the electron temperature, over the duration of the phonon pulse is well resolved. The layout of this paper is as follows. In section two a brief review of the properties of electrons in the fractional quantum Hall state and the phonons in the substrate is given, more details can be found in [4] and [5]. In section 3 expressions for the rate of energy transfer between a phonon pulse and the electron system will be given and the forms of the relevant matrix elements discussed. In sections four a simple analytical treatment of these rate expressions will be given which shows that magnetoroton dissociation due to 
heating by low energy phonons cannot occur. In section 5 the contribution of the magnetoroton scattering processes to the rate of energy transfer from the pulse to the 2des will be considered and it will be shown that there is a (low) cross-over temperature at which the dependence of the transfer rate on heater temperature changes from exponential to a power law. Section 6 will contain a brief summary and discussion.

\section{Electrons and Phonons in Fractional Quan- tum Hall Systems}

Our basic picture of the physics of electrons in the fractional quantum Hall states emerges from the ideas of Laughlin [8] and Girvin, MacDonald and Platzman [4. Laughlin constructed the wave function for a state with exceptionally low energy at the primary quantum Hall filling factor $\nu=1 /(2 m+1)$. He also constructed low energy quasiparticle excitations which carry fractional charge and have an energy gap even in the thermodynamic limit. Hence the description of the quantum Hall state as an incompressible quantum liquid. Girvin, MacDonald and Platzman [ 团 proposed wave functions for a branch of neutral collective excitations whose energy lies below that of the quasiparticle continuum. This situation is analogous to the phonon-roton branch in superfluid helium. GMP made this analogy more explicit by adapting the theoretical approach of Feynman [9] to the case of magnetically quantized $2 \mathrm{~d}$ electrons. They found a branch of low energy excitations whose dispersion relation, $\Delta(q)$, has a deep minimum at a finite wave vector, $q^{*}$. By analogy with liquid helium they dubbed these magnetorotons. Unlike helium, the fractional quantum Hall liquid does not have a gapless phonon mode at long wavelengths, the incompressibility of the underlying state causes the energy of the collective mode to increase for wavelengths longer than the magnetoroton minimum and to always be gapped. One expects the single mode approximation of GMP to give a good quantitative account of the physics of this collective mode for wavelengths close to and longer than the magnetoroton minimum. However, for shorter wavelengths one expects the mode to be better described as an exciton composed of a pair of oppositely charged Laughlin quasiparticles. The separation of the pair in a state of wave vector $q$ being $l_{c}^{2} q / \nu$. The form of the dispersion relation which we assume here is

$$
\Delta(q)=\left\{\begin{array}{cc}
\Delta_{\mathrm{SMA}}(q) & q<q_{m} \\
\Delta_{\infty}-E_{c} \frac{\nu^{3}}{q} & q>q_{m}
\end{array}\right.
$$

where $E_{c}=e^{2} / 4 \pi \varepsilon_{0} \kappa l_{c}$ is the Coulomb energy scale, $\Delta_{\mathrm{SMA}}$ is the dispersion relation obtained from the single mode approximation of GMP [4] and $q_{m}$ and $\Delta_{\infty}$ are chosen so that $\Delta(q)$ and its first derivative are continuous for all $q$. This is shown in figure 1 below. As can be seen the "exciton" part of this dispersion relation is very flat: a fact that will have considerable significance for the proposed dissociation mechanism. 
Phonons in a GaAs substrate travel ballistically for frequencies up to about a terahertz. Hence a pulse of phonons injected into a GaAs substrate will move across the substrate of a heterostructure until it encounters the heterojunction where it will interact with the vertically confined electrons. The Hamiltonian for the electron phonon interaction can be written in the form

$$
H_{e \phi}=\sum_{s, \underline{Q}} M_{s}(\underline{Q}) Z\left(q_{z}\right) \widehat{\rho}_{\mathbf{q}}\left(\widehat{a}_{s}(\underline{Q})+\widehat{a}_{s}^{\dagger}(-\underline{Q})\right)
$$

where $\widehat{\rho}_{\mathbf{q}}$ is the Fourier transform of the local (2d) electron density, $\widehat{a}_{s}(Q)$ is the second quantized operator for phonons of polarization $s$ and (3d) wave vector $\underline{Q}, Z\left(q_{z}\right)$ is a form factor to account for the finite thickness of the electron layer and $M_{s}(\underline{Q})$ is the matrix element for the coupling of phonons in the given mode to electrons close to the $\Gamma$ point of the GaAs conduction band. This hamiltonian incorporates both piezoelectric and deformation potential coupling which differ in their anisotropy and in the index of the power law dependence on phonon energy.

The trial wave function used by GMP for a magnetoroton of wave vector $\mathbf{q}$ is of the form

$$
|\mathbf{q}\rangle=\frac{\bar{\rho}_{\mathbf{q}}\left|\Psi_{L}\right\rangle}{\sqrt{\left\langle\Psi_{L}\left|\bar{\rho}_{-\mathbf{q}} \bar{\rho}_{\mathbf{q}}\right| \Psi_{L}\right\rangle}}
$$

where $\left|\Psi_{L}\right\rangle$ is the Laughlin ground state and $\bar{\rho}_{\mathbf{q}}$ is the projection of the electron density operator onto the lowest Landau level: $\bar{\rho}_{\mathbf{q}}=\mathcal{P}_{0} \rho_{\mathbf{q}} \mathcal{P}_{0}$ (see [10] for details of the projection). If we consider a pulse of phonons characterized by a distribution function $n_{s}(\underline{Q})$ in contact with a 2 des in the fractional quantum Hall state at zero temperature, then the rate of energy transfer to the electrons should be, using Fermi's golden rule

$$
\begin{aligned}
P & =2 \pi \sum_{s, Q, \mathbf{k}}\left|M_{s}(\underline{Q}) Z\left(q_{z}\right)\left\langle\mathbf{q}\left|\widehat{\rho}_{\mathbf{q}}\right| \Psi_{L}\right\rangle\right|^{2} \delta\left(\omega_{s}(\underline{Q})-\Delta(\mathbf{k})\right) n_{s}(\underline{Q}) \\
& =2 \pi N \sum_{s, \underline{Q}, \mathbf{k}}\left|M_{s}(\underline{Q}) Z\left(q_{z}\right)\right|^{2} \bar{s}(q) \delta\left(\omega_{s}(\underline{Q})-\Delta(\mathbf{k})\right) n_{s}(\underline{Q})
\end{aligned}
$$

where

$$
\bar{s}(q)=\frac{1}{N}\left\langle\Psi_{L}\left|\bar{\rho}_{-\mathbf{q}} \bar{\rho}_{\mathbf{q}}\right| \Psi_{L}\right\rangle
$$

is the projected static structure factor of the electron liquid and we set $\hbar=l_{c}=1$ from now on. The projected structure factor is related, as shown by GMP, to the full structure factor, $s(q)$ by

$$
\bar{s}(q)-e^{-q^{2} / 2}=s(q)-1 \quad .
$$

GMP extracted $s(q)$ from Laughlin's wave function and used it in the calculation of the magnetoroton dispersion $\Delta(q)$. The expression in equation 5 forms the basis for the work in [5]. 


\section{Magnetoroton-Phonon Scattering}

Let us now consider the probability per unit time that a magnetoroton of wave vector $\mathbf{k}$ will absorb a phonon with wave vector $Q=\left(\mathbf{q}, q_{z}\right)$ and be scattered into the state with wave vector $\mathbf{k}+\mathbf{q}$. Fermi's golden rule tells us that this will be

$$
\tau_{\mathbf{k}, \underline{Q}}^{-1}=2 \pi\left|M_{s}(\underline{Q}) Z\left(q_{z}\right)\right|^{2}\left|\left\langle\mathbf{k}+\mathbf{q}\left|\rho_{\mathbf{q}}\right| \mathbf{k}\right\rangle\right|^{2} \delta\left(\Delta(k)+\omega_{s}(\underline{Q})-\Delta(|\mathbf{k}+\mathbf{q}|)\right)
$$

which we can express, using the ansatz of GMP, as

$$
2 \pi \frac{\left|M_{s}(\underline{Q}) Z\left(q_{z}\right)\right|^{2}}{\bar{s}(k) \bar{s}(|\mathbf{k}+\mathbf{q}|)}\left|P^{(3)}(\mathbf{k}, \mathbf{q})\right|^{2} \delta\left(\Delta(k)+\omega_{s}(\underline{Q})-\Delta(|\mathbf{k}+\mathbf{q}|)\right)
$$

where we define the projected three-point correlation function

$$
P^{(3)}(\mathbf{k}, \mathbf{q})=\frac{1}{N}\left\langle\Psi_{L}\left|\bar{\rho}_{-\mathbf{k}-\mathbf{q}} \bar{\rho}_{\mathbf{q}} \bar{\rho}_{\mathbf{k}}\right| \Psi_{L}\right\rangle \quad .
$$

The projected three-point correlation function has been discussed by MacDonald et al. [11] in the context of magnetoroton scattering by quenched impurities. As outlined there, this quantity can be related to its unprojected counterpart via

$$
\begin{aligned}
P^{(3)}(\mathbf{k}, \mathbf{q}) & =e^{-\frac{1}{2} q^{2}-\frac{1}{2}(\mathbf{k}, \mathbf{q})}\left\{e^{-\frac{1}{2} k^{2}}+s(k)-1\right\}+e^{-\frac{1}{2}((\mathbf{k}+\mathbf{q}), \mathbf{k})}(s(q)-1) \\
& +e^{\frac{1}{2}(\mathbf{q}, \mathbf{k})}(s(|\mathbf{k}+\mathbf{q}|)-1)+n^{(3)}(\mathbf{k}, \mathbf{q})
\end{aligned}
$$

where

$$
\begin{aligned}
(\mathbf{k}, \mathbf{q}) & =\mathbf{k} \cdot \mathbf{q}+i \mathbf{k} \wedge \mathbf{q} \\
& =\left(k_{x}-i k_{y}\right)\left(q_{x}+i q_{y}\right)
\end{aligned}
$$

and

$$
n^{(3)}(\mathbf{k}, \mathbf{q})=\frac{1}{N}\left\langle\Psi_{L}\left|\rho_{-\mathbf{k}-\mathbf{q}} \rho_{\mathbf{q}} \rho_{\mathbf{k}}\right| \Psi_{L}\right\rangle \quad .
$$

MacDonald et al. used the convolution approximation [12] developed for the study of corresponding processes in superfluid helium to estimate this latter quantity in terms of the static structure factor and obtained an expression for $n^{(3)}(\mathbf{k}, \mathbf{q})$ of the form

$$
\begin{aligned}
n^{(3)}(\mathbf{k}, \mathbf{q}) & =N^{2} \delta_{\mathbf{k}, \mathbf{0}} \delta_{\mathbf{q}, \mathbf{0}}+N \delta_{\mathbf{k}, \mathbf{0}} h(q)+N \delta_{\mathbf{q}, \mathbf{0}} h(k)+N \delta_{\mathbf{k}+\mathbf{q}, \mathbf{0}} h(k) \\
& +h(k) h(q)+[h(k)+h(q)+h(k) h(q)] h(|\mathbf{k}+\mathbf{q}|)
\end{aligned}
$$

where

$$
h(k)=s(k)-1-N \delta_{\mathbf{k}, 0} \quad .
$$


Beyond this, very little is known about this function, although a useful sum-rule for testing approximation schemes for it was recently derived in [13]. Although more detailed knowledge of this quantity would be desirable for truly quantitative work on the phonon experiments, here we will restrict ourselves to the more modest objective of qualitative understanding.

\section{Analytic Estimates}

We will begin by deriving a simple criterion for small momentum transfer magnetoroton scattering processes. Energy and momentum conservation require that a magnetoroton with initial wave vector $\mathbf{k}$ can absorb a phonon with in-plane momentum $\mathbf{q}$ incident at angle $\theta$ to the normal to the 2 des only when

$$
\Delta(|\mathbf{k}+\mathbf{q}|)=\Delta(k)+\frac{c_{s} q}{\sin \theta} .
$$

For small $q$ we set

$$
\Delta(|\mathbf{k}+\mathbf{q}|) \approx \Delta(k)+\frac{\mathbf{q} \cdot \mathbf{k}}{k} \Delta^{\prime}(k)
$$

so that

$$
\frac{\mathbf{q} \cdot \mathbf{k}}{k q}=\cos \phi=\frac{c_{s}}{\Delta^{\prime}(k) \sin \theta}
$$

where $\cos \phi=\mathbf{k} \cdot \mathbf{q} / k q$. This can only be satisfied when

$$
\left|\Delta^{\prime}(k)\right|>\frac{c_{s}}{\sin \theta}
$$

This result simply means that phonon absorption can only occur when the slope of the magnetoroton dispersion is greater than the slope of the dispersion curve for phonons with an angle of incidence $\theta$. By happy accident in our system of units $\left(\hbar=l_{c}=\mathcal{E}_{c}=1\right)$ the unit of velocity, $l_{c} \mathcal{E}_{0} / \hbar$, is independent of magnetic field and, for GaAs, has the value $1.68 \times 10^{5} \mathrm{~m} \mathrm{~s}^{-1}$. In these units the speeds of sound are $c_{1} \approx 0.0305$ for LA phonons and $c_{2}=c_{3} \approx 0.0196$ for TA phonons. Hence we expect that these scattering processes will be suppressed at large $k$ and sufficiently close to the magnetoroton minimum where the magnetoroton dispersion is very flat and $\Delta^{\prime}(k)$ small.

Let us estimate where this suppression occurs for large $k$ to assess the viability of the magnetoroton dissociation idea. At large $k$ we assume

$$
\Delta(k)=\Delta_{\infty}-\frac{\nu^{3}}{k}
$$

so that

$$
\Delta^{\prime}(k)=\frac{\nu^{3}}{k^{2}}
$$


and scattering will be cut-off beyond

$$
k_{s}=\sqrt{\frac{\nu^{3} \sin \theta}{c_{s}}} .
$$

Hence we have, for $\nu=1 / 3, l_{c} k_{1}=1.102, l_{c} k_{2}=1.375$, assuming that phonons with $\theta=\pi / 2$ (i.e. in plane phonons) exist in the pulse. The latter cut-off is only just beyond the magnetoroton minimum while the former is so small that the whole analysis is entirely meaningless. In fact, in the experiments, phonons with $\theta=\pi / 2$ are strongly focussed away from the device: only phonons with $\theta<\pi / 4$ are likely to couple with the electrons ensuring that there will be no coupling to very low energy phonons at all in the "exciton" regime. The corrections to this result arising from finite values of the phonon wave vector only strengthen it: the curvature of the magneto-exciton dispersion curve makes it even harder for energy and momentum to be conserved.

Let us now consider processes in which magnetorotons with wave vectors close to the minimum gap are scattered to longer wave lengths by absorbing phonons. In this case we have

$$
\Delta(k) \sim \Delta^{*}+\frac{\left(k-q^{*}\right)^{2}}{2 \mu}
$$

where $\mu$ is an effective magnetoroton mass. In this case the absorption of a small phonon with in-plane wave vector $q$ will be allowed provided

$$
\left|k-q^{*}\right|>\frac{\mu c_{s}}{\sin \theta}
$$

this gives, for $\nu=1 / 3$ (assuming the ideal magnetoroton dispersion) $\left|k-q^{*}\right|>$ $0.19 / l_{c}$ for LA phonons and $\left|k-q^{*}\right|>0.12 / l_{c}$ for TA phonons.

\section{Magnetoroton Scattering Contribution to Phonon Absorption}

The leading correction to the energy transfer rate due to magnetoroton creation at finite electron temperature will be due to the processes discussed above in which a magnetoroton is scattered from one mode to another with the absorption of a phonon. This contribution to the energy transfer rate will be given by

$$
\delta P=\sum_{s, \underline{Q}, \mathbf{k}^{\prime}} \omega_{s}(\underline{Q}) \tau_{\mathbf{k}^{\prime}, \mathbf{s}, \underline{Q}}^{-1} n_{B}\left(\omega_{s}(\underline{Q}) / T_{\phi}\right) n_{B}\left(\Delta\left(k^{\prime}\right) / T_{e}\right)
$$

where $T_{\phi}$ is the characteristic temperature of the phonon pulse and $n_{B}(x)=$ $\left(e^{x}-1\right)^{-1}$ is the Bose distribution function and we assume that $T_{e} \ll T_{\phi} \ll \Delta^{*}$ so that $n_{B}\left(\Delta(k) / T_{e}\right) \ll 1 \forall k$. In order to simplify matters we will neglect the finite thickness of the 2 d layer (i.e. we set $Z\left(q_{z}\right)=1$ ) and neglect the anisotropy 
in the electron phonon interaction (which at long wavelengths will be dominated by the piezoelectric coupling) and set

$$
M_{s}(\underline{Q})=\frac{\Lambda_{s}}{\sqrt{Q}}
$$

(strictly this bare piezoelectric coupling will be screened by the equilibrium population of excitations but we will not attempt to estimate such effects here). Finally we will use the isotropic Debye approximation (and hence neglect phononfocussing effects) setting

$$
\omega_{s}(\underline{Q})=c_{s} Q \quad .
$$

In the regime $T_{e} \ll T_{\phi} \ll \Delta^{*}$ the transfer of energy will be dominated by absorption by magnetorotons initially at the minimum, $k=q^{*} \approx 1.34 / l_{c}$, hence we will have

$$
\begin{aligned}
\delta P & \sim \sqrt{2 \pi \mu T_{e}} e^{-\Delta^{*} / T_{e}} \sum_{s} c_{s} \Lambda_{s}^{2} \int \frac{d^{3} \underline{Q}}{8 \pi^{3}} \frac{\left|P^{(3)}\left(q^{*} \widehat{\mathbf{n}}, \mathbf{q}\right)\right|^{2}}{\bar{s}\left(q^{*}\right) \bar{s}\left(\left|q^{*} \widehat{\mathbf{n}}+\mathbf{q}\right|\right)} \delta\left(\Delta\left(\left|q^{*} \widehat{\mathbf{n}}+\mathbf{q}\right|\right)-\Delta^{*}-c_{s} Q\right) \\
& \times n_{B}\left(c_{s} Q / T_{\phi}\right)
\end{aligned}
$$

where $\widehat{\mathbf{n}}$ is an arbitrary vector in the plane. Following [5] we transform to new variables to integrate out the delta-function and arrive at

$$
\begin{aligned}
\delta P & \sim \sqrt{\frac{\mu T_{e}}{2 \pi}} e^{-\Delta^{*} / T_{e}} \sum_{s} \Lambda_{s}^{2} \int \frac{d^{2} \mathbf{k}}{4 \pi^{2}} \frac{\omega_{k} \vartheta\left(\omega_{k}-c_{s}\left|\mathbf{k}-q^{*} \widehat{\mathbf{n}}\right|\right)}{\sqrt{\omega_{k}^{2}-c_{s}^{2}\left|\mathbf{k}-q^{*} \widehat{\mathbf{n}}\right|^{2}}} \\
& \times \frac{\left|P^{(3)}\left(q^{*} \widehat{\mathbf{n}}, \mathbf{k}-q^{*} \widehat{\mathbf{n}}\right)\right|^{2}}{\bar{s}\left(q^{*}\right) \bar{s}(k)} n_{B}\left(\omega_{k} / T_{\phi}\right)
\end{aligned}
$$

where the integration is now over all allowed transitions from the initial state $\left|q^{*} \widehat{\mathbf{n}}\right\rangle$ to a final state $|\mathbf{k}\rangle$ and

$$
\omega_{k}=\Delta(k)-\Delta^{*}
$$

is the corresponding energy change. The effective transition rate is simply understood by using a polar representation for the $\mathbf{k}$-plane, with $\widehat{\mathbf{n}}$ as the polar axis. The scatering rate is then

$$
\begin{aligned}
\tau_{\mathbf{k}}^{-1} & =\frac{\vartheta\left(\omega_{k}^{2}-c_{s}^{2}\left(k^{2}+q^{* 2}-2 q^{*} k \cos \phi\right)\right)}{\sqrt{\omega_{k}^{2}-c_{s}^{2}\left(k^{2}+q^{* 2}-2 q^{*} k \cos \phi\right)}} \Phi(k, \phi) \\
\Phi(k, \phi) & =\frac{\left|P^{(3)}\left(q^{*} \widehat{\mathbf{n}}, \mathbf{k}-q^{*} \widehat{\mathbf{n}}\right)\right|^{2}}{\bar{s}\left(q^{*}\right) \bar{s}(k)} .
\end{aligned}
$$

Numerical study, using the form given above for $\Delta(k)$ (equation 1i) and the speed of sound, $c_{s}$, appropriate for TA phonons, shows that the condition imposed 
by the theta-function is only satisfied for final states within a contour $k_{c}(\phi)$ (shown in figure 2) in the k-plane, confirming the simple analysis presented in the previous section. Only the states inside this contour are accessible from the initial state $\left|q^{*} \widehat{\mathbf{n}}\right\rangle$. Along any direction in the $\mathbf{k}$-plane, there is a square root singularity as $k \rightarrow k_{c}(\phi)$ which will dominate the rate of energy transfer. The form of $\tau^{-1}$ along several representative directions in the $\mathbf{k}$-plane are shown in figure 3 . In this case we have used the simple ansatz for $P^{(3)}$ proposed by MacDonald et al. 11

$$
P^{(3)}(\mathbf{k}, \mathbf{q}) \approx 2 i \sin \left(\frac{1}{2}|\mathbf{k} \times \mathbf{q}|\right)
$$

although results obtained from the convolution approximation (equations 11 and 14) are qualitatively similar. In the appendix below, the behaviour of $\delta P$ is considered further and it is shown that for a given $\phi$ there is a characteristic temperature $T_{X}(\phi)=\Delta(k(\phi))-\Delta^{*}$ above which the dependence on heater temperature, $T_{\phi}$, develops a non-activated component.

It is clear then, that a non-activated component will appear in $\delta P$ when $T_{\phi}>\inf _{\phi}\left(T_{X}(\phi)\right)=T_{X}(0)$. To give an idea of the physical orders of magnitude involved, consider a 2 des with sheet density $n_{s}=10^{15} \mathrm{~m}^{-2}$. The $\nu=1 / 3$ fractional quantum Hall state will occur at a field $B=12.4 \mathrm{~T}$. The cyclotron length is then $l_{c}=\sqrt{\hbar / e B}=7.3 \mathrm{~nm}$ and the Coulomb energy scale is $\mathcal{E}_{c}=$ $e^{2} / 4 \pi \epsilon_{0} \kappa l_{c}=15.2 \mathrm{meV}$ (assuming $\kappa=13$ appropriate for GaAs). Using the form of the magnetoroton dispersion derived by GMP [ 1 gives $\Delta^{*} \simeq 0.075 \mathcal{E}_{c}=$ $1.14 \mathrm{meV}$ which corresponds to a temperature of $13 \mathrm{~K}$ and $q^{*} \simeq 1.3 l_{c}^{-1}=1.8 \times$ $10^{8} \mathrm{~m}^{-1}$. Using this dispersion relation we find, for TA phonons, $k(0)=1.08 / l_{c}$ which gives $T_{X}(0)=\Delta(k(0))-\Delta^{*}=0.005 \mathcal{E}_{c}=0.067 \Delta^{*}$ corresponding to a temperature of $870 \mathrm{mK}$. Given that the value of the gap extracted from the phonon experiments (which is reduced from the ideal value by the combined effects of disorder, finite thickness of the 2 des and Landau level mixing) is more like $4 \mathrm{~K}$ we might expect $T_{X}(0)<200 \mathrm{mK}$ : well below the heater temperature used in any of the experiments (and close to the base temperature of the dilution refrigerator). Hence we deduce that there will always be a contribution to the energy transfer rate which is non-exponential in the heater temperature (although its dependence of the electron temperature is still of the form $e^{-\Delta^{*} / T_{e}}$.

\section{Conclusions}

In this paper we have considered processes in which magnetorotons existing at finite electron temperature $T_{e}$ are scattered by absorbing phonons from a pulse characterized by a phonon temperature $T_{\phi}$. We have shown that, contrary to our earlier supposition [7], such processes will not heat the magnetorotons to sufficiently large wave vectors that they dissociate into separate charged quasiparticles and so directly contribute to the longitudinal conductivity. This negative result is important in that the phonon absorption experiments [2] [3] measure the change in longitudinal conductivity as a ballistic phonon pulse hits 
the 2des. The absence of any direct dissociation mechanisms means that the energy transferred to the 2 des is thermalized within the 2 des, raising its effective temperature and hence leading to an increase in the thermal population of charged quasiparticles. Consequently, the changes in $\sigma_{x x}$ can be calibrated against equilibrium magneto-transport experiments to give a direct measure of the non-equilibrium electron temperature.

Although the processes discussed can not dissociate the magnetorotons into charged quasiparticles, scattering to longer wavelengths is possible and will give a contribution to the energy transfer rate which is exponential in the electron temperature but will have an non-exponential form except at the very lowest heater temperatures. Such a power law dependence (on $T-T_{X}(0)$ ) might well be observable in current phonon absorption experiments performed over a range of base electron temperatures. It has recently been shown 14 that the lowest lying neutral excitation at long wavelengths is not the small $q$ magnetoroton but a bound state of two magnetorotons. It is then possible that the absorption of energy from a higher temperature phonon pulse may involve a cascade process in which magnetorotons are scattered to long wavelengths by absorption of low energy phonons and then decay into two roton bound states which are thermally dissociated, leading to an enhanced population of magnetorotons at the minimum.

Acknowledgement 1 This work was supported by the EPSRC (UK). We would like to acknowledge many useful discussions on this topic with CJ Mellor, $U$ Zeitler, S. Roshko, A. Devitt and J. Digby.

\section{A Analysis of energy transfer rate}

We have the following form for the scattering contribution to the energy transfer rate

$\delta P \sim \frac{1}{4 \pi^{2}} \sqrt{\frac{\mu T_{e}}{2 \pi}} e^{-\Delta^{*} / T_{e}} \sum_{s} \Lambda_{s}^{2} \int_{0}^{2 \pi} d \phi Y(\phi) \int_{0}^{k(\phi)} d k \frac{\vartheta\left(\omega_{\mathbf{k}}-c_{s}\left|\mathbf{k}-q^{*} \widehat{\mathbf{n}}\right|\right)}{\sqrt{\omega_{k}^{2}-c_{s}^{2}\left|\mathbf{k}-q^{*} \widehat{\mathbf{n}}\right|^{2}}} n_{B}\left(\omega_{k} / T_{\phi}\right)$

where

$$
Y(\phi)=k(\phi) \omega(k(\phi)) \frac{\left|P^{(3)}\left(q^{*} \widehat{\mathbf{n}}, \mathbf{k}(\phi)-q^{*} \widehat{\mathbf{n}}\right)\right|^{2}}{\bar{s}\left(q^{*}\right) \bar{s}(k(\phi))}
$$

and

$$
\mathbf{k}(\phi)=k(\phi)(\cos \phi \widehat{\mathbf{n}}+\sin \phi \widehat{\phi})
$$


so that

$$
\begin{aligned}
\delta P & \sim \frac{1}{4 \pi^{2}} \sqrt{\frac{\mu T_{e}}{2 \pi}} e^{-\Delta^{*} / T_{e}} \sum_{s} \Lambda_{s}^{2} \int_{0}^{2 \pi} d \phi \frac{Y(\phi)}{\sqrt{|Q(\phi)|}} \\
& \times \int_{0}^{k(\phi)} \frac{d k}{\sqrt{k(\phi)-k}} n_{B}\left(\frac{\Delta(k(\phi))-\Delta^{*}+(k(\phi)-k)\left|\Delta^{\prime}(k(\phi))\right|}{T_{\phi}}\right)
\end{aligned}
$$

where

$$
Q(\phi)=2\left[c_{s}^{2}\left(k(\phi)-q^{*} \cos \phi\right)-\Delta^{\prime}(k(\phi))\left(\Delta(k(\phi))-\Delta^{*}\right)\right] \quad .
$$

Now we define

$$
\begin{aligned}
a(\phi) & =\Delta(k(\phi))-\Delta^{*} \\
b(\phi) & =\left|\Delta^{\prime}(k(\phi))\right| \\
\tau & =T_{\phi} / a(\phi)
\end{aligned}
$$

so that we need to consider the integral

$$
\int_{0}^{\infty} \frac{d \eta}{\sqrt{\eta}} \frac{1}{e^{(a+b \eta) / a \tau}-1}=\frac{T_{\phi}}{\sqrt{a b}} \int_{1 / \tau}^{\infty} \frac{d x}{\sqrt{\tau x-1}\left(e^{x}-1\right)} .
$$

We will use the an interpolation formula for the Bose function

$$
n_{B}(x)= \begin{cases}1 / x & x<1 \\ e^{-x} & x>1\end{cases}
$$

and consider two cases. Firstly, in the case that $\tau<1$ we have that

$$
\begin{aligned}
\int_{1 / \tau}^{\infty} \frac{d x}{\sqrt{\tau x-1}\left(e^{x}-1\right)} & \sim \int_{1 / \tau}^{\infty} \frac{e^{-x} d x}{\sqrt{\tau x-1}} \\
& =\sqrt{\frac{\pi}{\tau}} e^{-1 / \tau}
\end{aligned}
$$

In the other case, we have

$$
\begin{aligned}
\int_{1 / \tau}^{\infty} \frac{d x}{\sqrt{\tau x-1}\left(e^{x}-1\right)} & \sim \int_{1 / \tau}^{1} \frac{d x}{x \sqrt{\tau x-1}}+\int_{1}^{\infty} \frac{e^{-x} d x}{\sqrt{\tau x-1}} \\
& =2 \arctan (\sqrt{\tau-1})+\sqrt{\frac{\pi}{\tau}} e^{-1 / \tau}\left(1-\operatorname{erf}\left(\frac{\sqrt{\tau-1}}{\sqrt{\tau}}\right)\right)
\end{aligned}
$$

Hence, for $\tau>1$ there is a contribution that is non-activated in the phonon temperature. The angular $(\phi)$ integration is non-trivial and its numerical evaluation is rather pointless given our neglect of anisotropy in phonon propagation and the electron-phonon coupling. It would however be expected, for $T_{\phi} \gtrsim T_{X}(0)$ to have a power law dependence on $T_{\phi}-T_{X}(0)$. 


\section{References}

[1] L.J. Challis and A.J. Kent, in "Aspects of Semiconductor Nanostructures" (ed. G. Bauer, F. Kuchar and H. Heinrich) (Heidelberg: Springer, 1992) p31.

[2] C.J. Mellor, R.H. Eyles, J.E. Digby, A.J. Kent, K.A. Benedict, L.J. Challis, M. Henini, C.T. Foxon, Phys. Rev. Lett. 74, 2339 (1995).

[3] U. Zeitler, A.M. Devitt, J.E. Digby, C.J. Mellor, A.J. Kent, K.A. Benedict, T. Cheng, Phys. Rev. Lett., 82, 5333 (1999).

[4] S.M. Girvin, A.H. MacDonald and P.M. Platzman, Phys Rev. B33, 2481 (1986).

[5] K.A. Benedict, R.K. Hills, C.J. Mellor, Phys. Rev. B 60, 10984 (1999).

[6] P.M. Platzman, Phys Rev B39, 7985 (1989).

[7] K.A. Benedict in "Proceedings of the Twelfth International Conference on High Magnetic Fields in the Physics of Semiconductors", (Singapore: World Scientific), 95 (1997).

[8] R.B. Laughlin, Phys Rev Lett. 50, 1395 (1983).

[9] R.P. Feynman, Phys Rev 91, 1291 (1953).

[10] S.M. Girvin and T. Jach, Phys Rev B 29, 5617 (1984).

[11] A.H. MacDonald, K.L. Liu, S.M. Girvin, P.M. Platzman, Phys. Rev. B33, 4014 (1986).

[12] H.W. Jackson and E. Feenberg, Rev. Mod. Phys. 34, 686 (1962).

[13] M. Brownlie and K.A. Benedict, J. Phys. A. 33, 4283 (2000).

[14] K. Park and J.K. Jain, Phys. Rev. Lett. 84, 5576 (2000).

\section{Figure Captions}

Figure 1: A plot of form of the magnetoroton dispersion $\Delta(\mathbf{q})$ used here.

Figure 2: A plot of the contour $k_{c}(\phi)$ in $\mathbf{k}$-space. Only magnetoroton states in the shaded region inside the contour are accessible from the initial state $\left|q^{*} \widehat{\mathbf{n}}\right\rangle$ via a single phonon scattering process.

Figure 3: Representative plots of the scattering rate into states with wavenumber $k$ for a sample set of directions, $\phi$. The square root singularities in each trace occur at the critical value $k_{c}(\phi)$. 


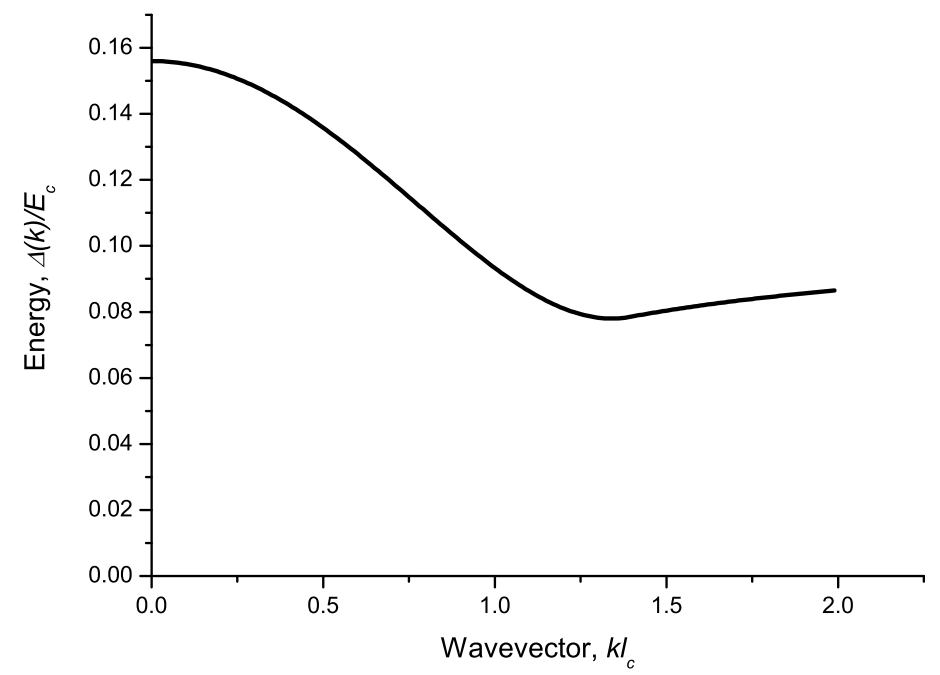




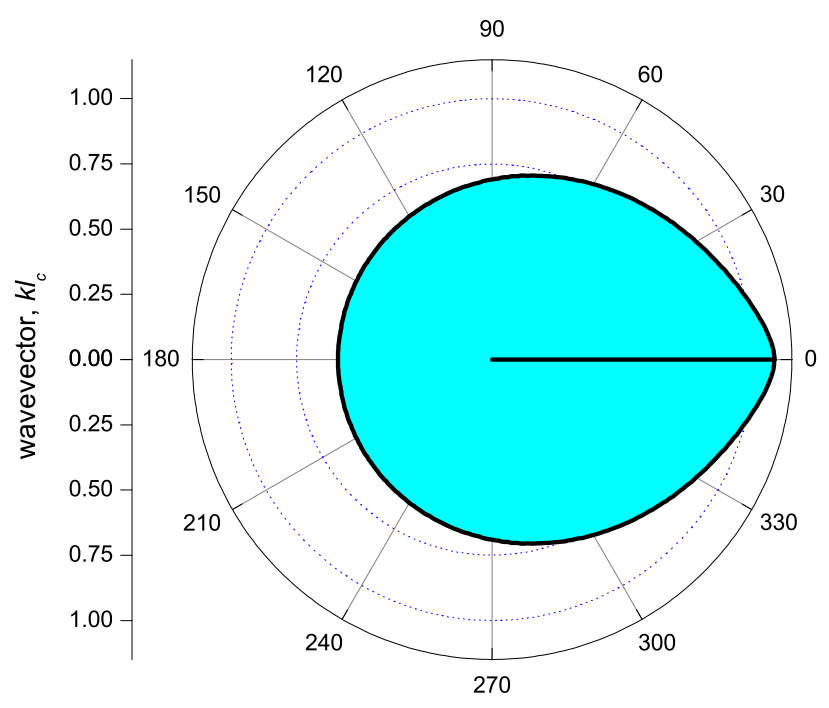




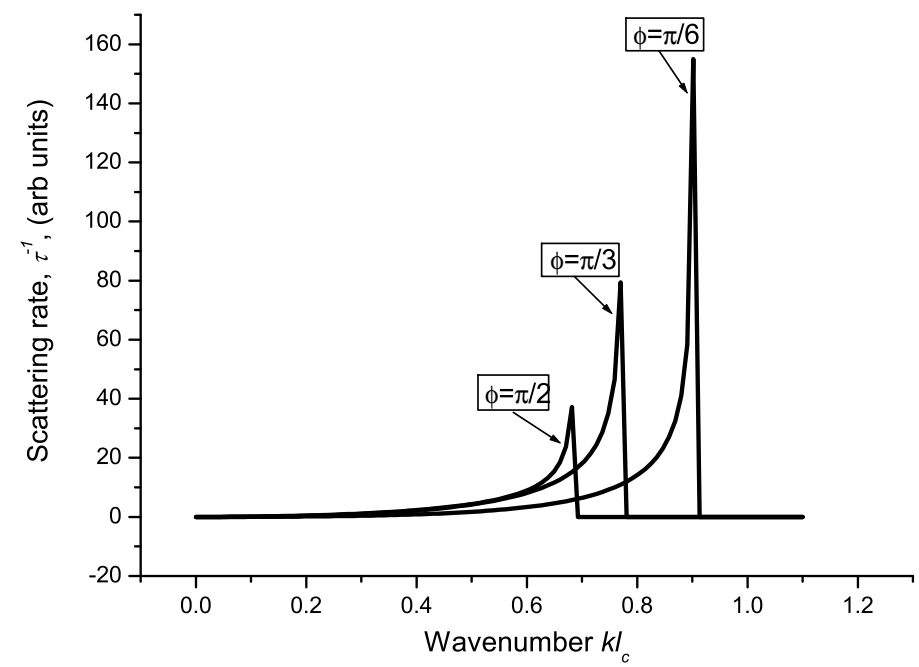

